Zoo Biology 18:53-62 (1999)

\title{
Social Interactions Between Captive Adult Male and Infant Lowland Gorillas: Implications Regarding Kin Selection and Zoo Management
}

\author{
A. Elizabeth Enciso, ${ }^{1}$ James M. Calcagno, ${ }^{2 \star}$ and Kenneth C. Gold ${ }^{3}$ \\ ${ }^{1}$ University of Michigan, Ann Arbor, Michigan \\ ${ }^{2}$ Loyola University of Chicago, Chicago, Illinois \\ ${ }^{3}$ Apenheul Primate Park, Apeldoorn, The Netherlands
}

\begin{abstract}
Interactions between unrelated and related silverback-infant dyads are compared in an attempt to assess the influence that kinship may have on male parental behavior. Observational data were collected on each member of two silverbackinfant dyads, in two separate enclosures at the Lincoln Park Zoo in Chicago, IL. The silverback was the father of the infant in one dyad, and unrelated to the infant in the other. Each infant was responsible for initiating most of the encounters with its respective group silverback. However, based on the frequency and duration of interactions, there is a significantly higher degree of affiliation and tolerance within the silverback-offspring dyad. Furthermore, the unrelated infant was the recipient of more than $40 \%$ of the agonistic behaviors exhibited by the silverback, whereas no such encounters were recorded within the related dyad. Although alternative explanations must be considered, these findings are consistent with kin selection theory, are similar to observations documented for wild mountain gorillas, and provide uncommon comparative data on adult male interactions with related and unrelated infants. In addition, this study offers behavioral information relevant to the management of captive gorillas, which often requires the introduction of immatures into non-natal groups. Zoo Biol 18:5362, 1999. ๑ 1999 Wiley-Liss, Inc.
\end{abstract}

Key words: Gorilla gorilla gorilla; silverback parental behavior; paternity confidence; male aggression; infant development

\section{INTRODUCTION}

Male parental behavior is highly variable throughout the primate order. Kin selection theory provides a possible explanation for such variation by partly attribut-

*Correspondence to: Dr. James M. Calcagno, Department of Sociology \& Anthropology, Loyola University of Chicago, 6525 N. Sheridan Road, Chicago, IL 60626. E-mail: jcalcag@luc.edu

Received for publication August 11, 1997; revision accepted November 30, 1998.

(C) 1999 Wiley-Liss, Inc. 
Enciso et al.

ing the degree of male investment to the level of "confidence of paternity" [Trivers, 1985]. Studies of wild mountain gorillas (Gorilla gorilla beringei) indicate a group structure in which there is usually one or more adult silverback males who restrict access of other males to reproductive females. Offspring conceived in the group are likely to have been sired by these dominant males [Harcourt et al., 1981; Watts, 1996]. Consequently, dominant males are expected to exhibit greater involvement with infants.

Observations from behavioral studies of wild mountain gorillas support a kin selection hypothesis, as dominant silverbacks tend to assume a parental strategy characterized by high levels of tolerance and affiliative interactions with group immatures [Fossey, 1979; Stewart and Harcourt, 1986; Whitten, 1986]. Initially, the infantsilverback association is primarily a result of the mother's efforts to remain in close proximity to a dominant male with her dependent offspring. However, as infants mature, maternal proximity time with a leading silverback decreases while infantsilverback proximity time increases [Harcourt, 1979], with infant interactions peaking between the third and fourth years [Fossey, 1979; Whitten, 1986]. Immatures are the primary initiators of such social encounters with an adult male, and they spend a considerable portion of feeding, resting, and play time in his proximity [Schaller, 1963; Fossey, 1979; Stewart and Harcourt, 1986; Watts and Pusey, 1993]. Indeed, such encounters often become more frequent than infant-mother interactions [Harcourt, 1979; Watts and Pusey, 1993].

Comparative data on infant-adult male interactions among lowland gorillas are not available due to the difficulty of conducting behavioral observations among these largely unhabituated groups [Tutin, 1996]. Although studies indicate similar social organizations of the subspecies, this is not sufficient for assuming similar patterns of social behavior [Tutin, 1996; Watts, 1996]. However, in their study of captive lowland gorillas (G. g. gorilla), Tilford and Nadler [1978] suggest that adult males show an even greater active interest in immatures than is typically seen among wild mountain gorillas. Thus, research on captive lowland gorillas provides an important avenue for exploring the nature of adult male parental behavior in primates.

In this study, interactions within unrelated and related silverback-infant dyads are analyzed in an attempt to assess the influence that kinship may have on male parental behavior. In addition, silverback associations with other group members are considered to assess their relative level of interactions with the infants. Importantly, possible confounding factors and alternative explanations, such as differences in personality, age, and rearing, are viewed in the context of our results. Both theoretical and practical implications of the data are addressed.

\section{METHODS Subjects}

The unrelated and related silverback-infant dyads observed in this study were members of two different groups of lowland gorillas housed in separate enclosures at the Lincoln Park Zoo in Chicago, IL (see Table 1 for information on group members). The infant member (Hasani) of the related dyad was born in the group, but he and his mother (Benga) were separated from the group shortly thereafter. Reintroduction of both mother and infant occurred 12 months later, and although possible, it is unlikely that the silverback (Gino) recognized the infant. Therefore, any signifi- 
Male Gorilla Parental Behavior

TABLE 1. Profiles of gorilla groups containing the related and unrelated dyads

\begin{tabular}{|c|c|c|c|c|c|c|c|}
\hline $\begin{array}{l}\text { Related } \\
\text { dyad }\end{array}$ & Name & Sex & $\begin{array}{c}\text { Age } \\
\text { during } \\
\text { study }\end{array}$ & $\begin{array}{c}\text { Unrelated } \\
\text { dyad }\end{array}$ & Name & Sex & $\begin{array}{c}\text { Age } \\
\text { during } \\
\text { study }\end{array}$ \\
\hline Silverback & Gino & M & 15 yrs & Silverback & Frank & M & 32 yrs \\
\hline Infant & Hasani & M & $19-21 \mathrm{mos}$ & Infant & M’Bizi & M & $33-35 \mathrm{mos}$ \\
\hline \multirow{8}{*}{$\begin{array}{c}\text { Other group } \\
\text { members }\end{array}$} & Benga & $\mathrm{F}$ & $25 \mathrm{yrs}$ & Other group & Mumbi & $\mathrm{F}$ & 35 yrs \\
\hline & Lenore & $\mathrm{F}$ & 33 yrs & members & Debbie & $\mathrm{F}$ & $30 \mathrm{yrs}$ \\
\hline & Helen & $\mathrm{F}$ & 38 yrs & & Kumba & $\mathrm{F}$ & $26 \mathrm{yrs}$ \\
\hline & Kowali & $\mathrm{F}$ & $18 \mathrm{yrs}$ & & Bulera & $\mathrm{F}$ & $7 \mathrm{yrs}$ \\
\hline & Hope & $\mathrm{F}$ & 12 yrs & & Bahati & $\mathrm{F}$ & 5 yrs \\
\hline & Makari & $\mathrm{F}$ & 9 yrs & & Zawadi & M & $4-5$ yrs \\
\hline & & & & & Tabibu & $\mathrm{F}$ & 4 yrs \\
\hline & & & & & Madini & $\mathrm{F}$ & $0-2$ yrs \\
\hline
\end{tabular}

cant level of paternity confidence rests on the silverback's previous association with the infant's mother. When the infant was born, his mother had been residing in the same group as the silverback for 6 years, and she had given birth to a female offspring (sired by Gino) 4 years earlier. Thus, a long-term association existed between Gino and Benga, which may have been a critical determinant of his level of involvement with the infant.

As with Hasani, the infant (M'Bizi) of the unrelated dyad was also separated from members of his natal group (including both parents). Beginning at 20 months of age, M'Bizi was temporarily housed with an adult female. However, unlike Hasani with his mother, M'Bizi was paired with an unrelated female (Debbie) of silverback Frank's group. Debbie served as the infant's "surrogate mother," partially due to her past success in such a role. After 3 months, M'Bizi, accompanied by Debbie, was introduced to Frank's group and has since resided there, apart from both of his biological parents. Prior to this introduction, M'Bizi had no previous association with Frank, and although "surrogate mother" Debbie had resided with Frank since infancy, she never bore any offspring due to reproductive health problems and had no reproductive history with Frank. Thus, some similarities do exist regarding the rearing of each infant, but contrary to the related dyad, the unrelated infant was not associated with his mother or a reproductive female, and there were no strong reasons to expect Frank to behave as if paternity confidence existed with M'Bizi.

\section{Analysis}

Observational data were collected using focal animal sampling [Altmann, 1974] on each member of the two silverback-infant units. Approximately $2.5 \mathrm{hr}$ of data were collected on each individual per week for 8 weeks during the summer of 1996. Thus, $40 \mathrm{hr}$ of data were accumulated for each dyad, totaling $80 \mathrm{hr}$ of observation.

All data were collected by the first author (A.E.E.) in 15-min intervals using The Observer 3.0 software. Behaviors were scored according to a behavioral ethogram [modeled after Bennett and Fried, 1991; DuBois et al., 1991; Gold, 1991, 1993; Hoff et al., 1981] accounting for solitary, abnormal, affiliative, and agonistic behaviors; although only the latter two categories of behaviors are considered here (Table 2). The initiator and recipient of affiliative and agonistic encounters were noted to identify the primary instigator of the interactions within each dyad. 
TABLE 2. Ethogram of affiliative and agonistic social behaviors recorded in study

\begin{tabular}{|c|c|}
\hline Proximity & Out of contact but within arm's reach of another gorilla (arm length of larger animal). \\
\hline Contact & Focal animal is in direct physical contact with another gorilla. \\
\hline Approach & Focal animal moves toward another to within proximity. \\
\hline Withdraw & One animal retreats from another as the result of an approach. \\
\hline Social play & $\begin{array}{l}\text { Physical play with a partner. This behavior includes chase, mock bite, poke/hit, throw at, } \\
\text { object tug, wrestle, etc. Does not necessitate continuously maintained proximity with } \\
\text { another play partner. }\end{array}$ \\
\hline Follow & $\begin{array}{l}\text { Focal animal independently locomotes behind another within a distance of two body } \\
\text { lengths in an effort to maintain or restore proximity. }\end{array}$ \\
\hline \multicolumn{2}{|c|}{ Agonistic Behaviors } \\
\hline Display & $\begin{array}{l}\text { Behaviors used by an animal that draw attention to that animal. May include tight-lip } \\
\text { stare, stiff stance, strut walk, chest beat, charge, hand clap, object/wall/ground slap, } \\
\text { or slam/bang door. }\end{array}$ \\
\hline Aggression & $\begin{array}{l}\text { Charge with hitting, hit object, chase, bit, throw objects, push, grab/pull hair, hit/slap, } \\
\text { poke, wrestle, roar. Distinguished from social play. }\end{array}$ \\
\hline Submission & $\begin{array}{l}\text { Turn away from, avoid/withdraw from, crouch/cower, present, hide, run away form, } \\
\text { scream. }\end{array}$ \\
\hline
\end{tabular}

Data were downloaded from The Observer into Quattro-Pro spreadsheets for the calculation of descriptive statistics and $t$-tests of statistical significance. Mean occurrences per hour were calculated by dividing the total number of occurrences of a behavior by the total number of hours that both members of a dyad were in sight. $t$ Tests were based on the total amount of time (80 15-min sessions for each dyad) for the entire study [Gold, 1993].

\section{RESULTS}

In both dyads, the infants were responsible for the majority of approaches (Fig. 1). Silverback-initiated approaches were infrequent, with no significant difference between dyads. However, the rate of infant approaches in the related dyad was significantly higher than that within the unrelated pair $(P<0.001)$, and the number and duration of social interactions following the initial approach reveals substantial differences between the two units. Proximity and contact occurrences were significantly higher in the related unit $(P<0.001)$, and the total amount of time spent in proximity (6.7 vs. $2.4 \mathrm{~min} / \mathrm{hr})$ and in contact ( $1.0 \mathrm{vs} .0 .2 \mathrm{~min} / \mathrm{hr}$ ) was even more disparate. Also, bouts of social play and incidents of the infant following the silverback were only observed in the adult male-offspring pair $(P<0.05$, Fig. 2$)$.

Although agonistic interactions were infrequent, differences between the two dyads are considerable (Fig. 2). In the related unit, there were no incidents of infant-directed aggression or display. Both, however, were observed between members of the unrelated unit $(P<0.05)$. Similarly, occurrences of infant submission, although statistically insignificant, were more than twice as frequent for the unrelated infant.

Silverback-group interactions were considered to determine what percentage of the silverback's social time comprised encounters with infants in comparison to other group members (Fig. 3). In the unrelated group, the infant accounted for $20.2 \%(2,061 / 10,211$ 


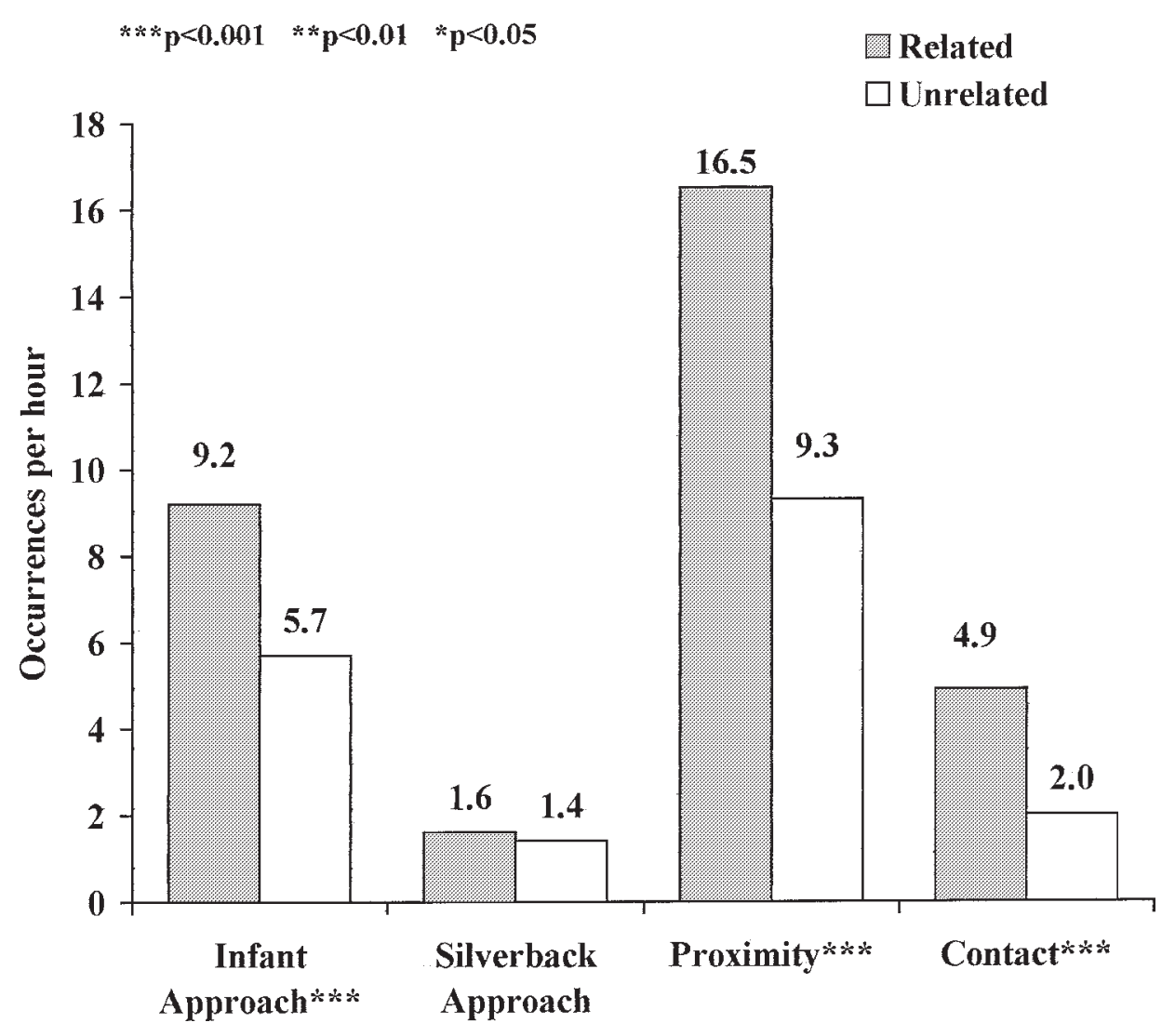

Fig. 1. Comparison of affiliative interactions within unrelated and related dyads.

sec) of the silverback's proximity time and $59.0 \%(155 / 263 \mathrm{sec})$ of his contact time. In contrast, the infant of the related pair represented $66.6 \%(8,539 / 12,820 \mathrm{sec})$ of the adult male's proximity time and $81.0 \%(1,319 / 1,628 \mathrm{sec})$ of his contact encounters. In fact, mother and infant accounted for $85.8 \%$ of proximity and $93.4 \%$ of contact bouts for the silverback. (Comparative data were impossible for the unrelated dyad, as the infant's mother does not reside in the group.) The total proximity time spent near a group member, excluding the infant, was nearly twice as great for the unrelated silverback $(8,150$ vs. $4,281 \mathrm{sec}$ ). Thus, total proximity times for each silverback are similar, yet the total duration time that Gino was in proximity to the related infant was more than four times greater than that of Frank and the unrelated infant. Similarly, the total duration of contact time was 8.5 times greater for Gino and the infant.

\section{DISCUSSION}

Similar to findings on wild mountain gorillas, the infants in this study were the primary initiators of social interactions with the dominant male. Both silverbacks in the unrelated and related units showed a low level of active interest in the infants in their respective group, as suggested by a frequency of silverback-initiated encounters that averaged less than two per hour within each dyad. 


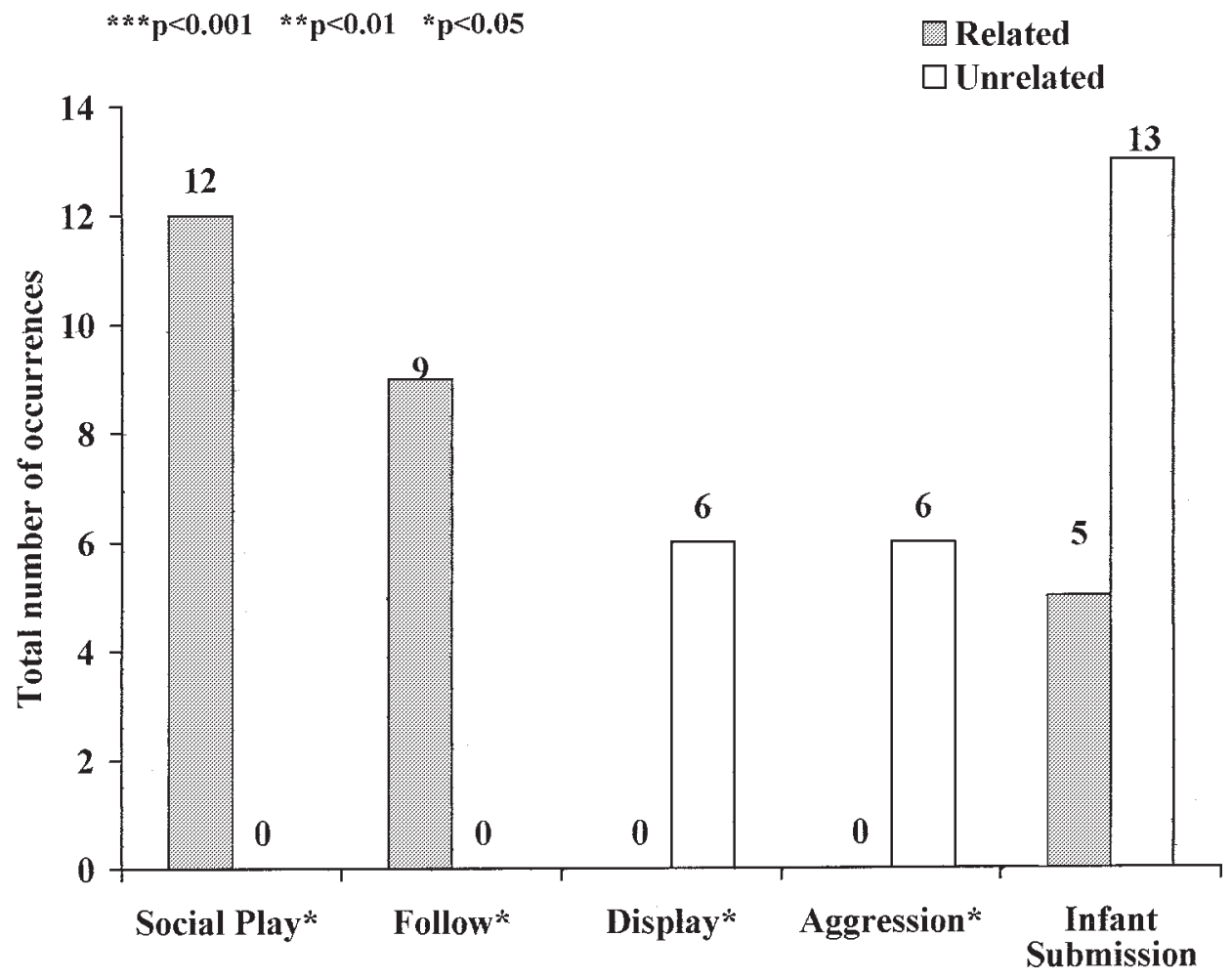

Fig. 2. Total number of occurrences of active affiliative (social play and follow) and agonistic behaviors in unrelated and related dyads during the entire study.

Although the silverbacks rarely initiated interactions with the infants, the related male reacted to the infant with a greater degree of tolerance and affiliative behavior. This behavioral difference is most strongly supported by proximity and contact occurrences that are roughly three and five times greater than those of the unrelated dyad (and even higher when judged by total duration time). In addition, only the unrelated silverback engaged in infant-directed displays and aggression. It is worth noting that, all else being equal, one might expect an older, more experienced silverback and a more mature infant to interact more [Harcourt, 1979; Fossey, 1979; Whitten, 1986; Watts and Pusey, 1993] , but this is the opposite of what is observed with the unrelated dyad. Also, although the unrelated silverback spent much less time in proximity to the infant, he was not isolated in general, but on the contrary had nearly twice the amount of proximity time with other group members than exhibited by the related silverback.

These results are in further agreement with those of wild studies of mountain gorillas in that parental investment by dominant male gorillas follows a pattern of tolerance and affiliation, but not of intensive caretaking [Stewart and Harcourt, 1986; Whitten, 1986]. Because such behavioral patterns were not strongly exhibited by the unrelated silverback, this study is consistent with the hypothesis that kinship may be a contributing factor to the degree of expressed paternal behavior. As previously discussed, and unlike the unrelated dyad, there appears to be a high level of paternity 


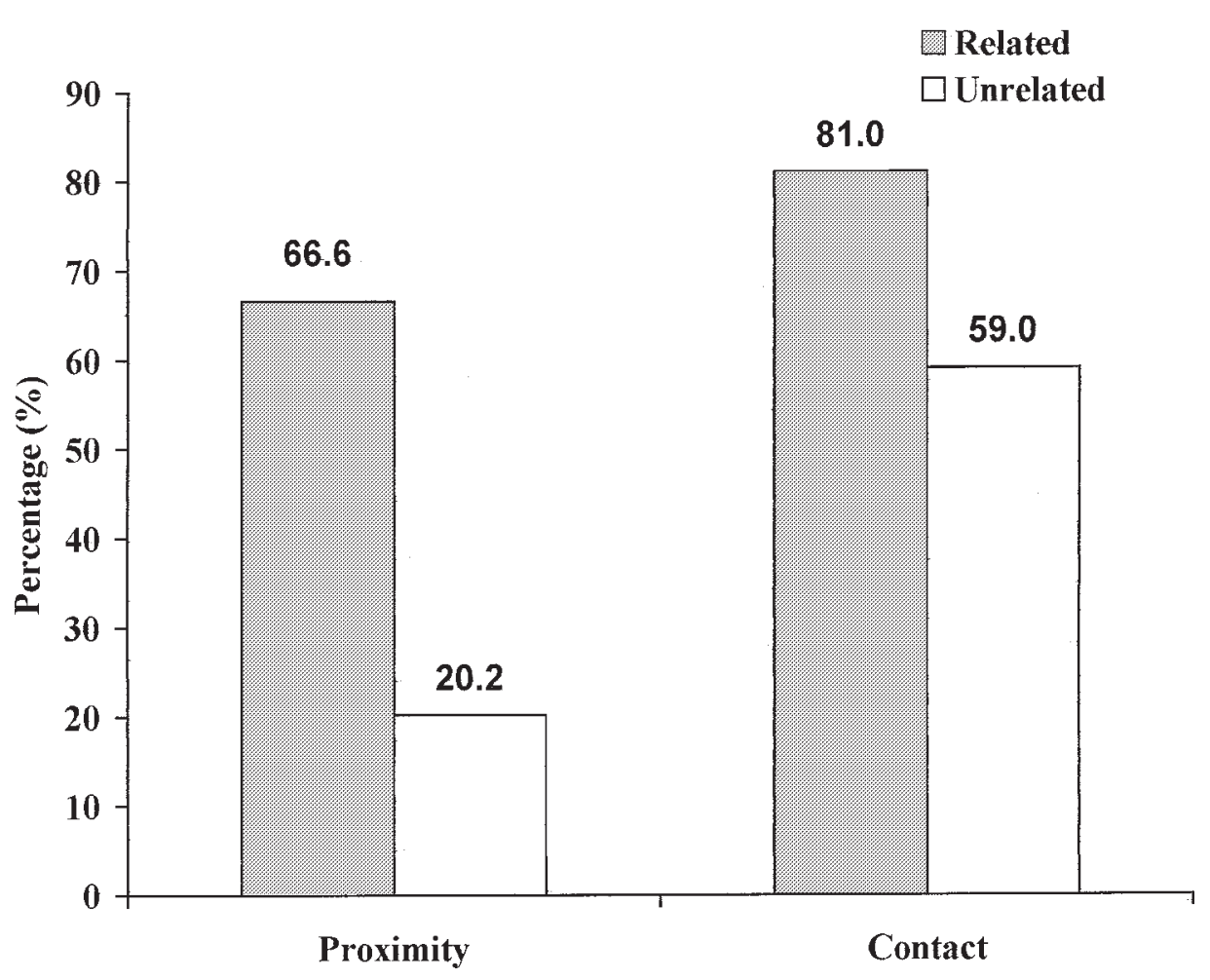

Fig. 3. Percentage of the total amount of silverback proximity and contact time, with all group members, that was spent with the infant.

confidence for the silverback of the related dyad, given his long-term association with the infant's mother. This interpretation is in agreement with the captive study by Tilford and Nadler [1978], who concluded that "the best predictor of the frequency of adult male-infant interactions was the affiliation bond between the adult male and each of the respective mothers." Similarly, in their observations of wild mountain gorillas, Harcourt and Stewart [1981] noted that a "friendly" relationship between the maturing gorilla and leading silverback was preceded by a "close" relationship (prolonged bouts of contact and proximity) between the mother-infant dyad and the dominant male. Conversely, in the absence of a previous bond with the mother and infant, leading males were observed to become increasingly less tolerant of the young gorilla, as also witnessed in the unrelated dyad of the current study.

Nevertheless, an explanation of the data based on kin selection rests on the assumption that the adult male's parental behaviors are primarily intended to be directed at offspring. It is possible, however, that such behavioral patterns may also serve indirectly to influence females. Harcourt [1979] suggests that, in the wild, silverback "baby-sitting" of infants may not only make protection of infants easier, but it may also influence a female's choice to stay in the group. Affiliation between adult males and infants could then be the result of a reproductive strategy used by the male to maintain his bond with the infant's mother, thereby ensuring future mating access [Whitten, 1986]. 
In addition, despite the consistency of the current results with kin selection theory, differences between the unrelated and related units may be attributable to a variety of possibly confounding variables, such as differences in group structure, personality, age, and rearing conditions. For example, the unrelated infant was housed with three other immature gorillas, which could have affected the lower frequencies of his social interactions with the silverback, due to the greater diversity of the infant's social environment. In contrast, the infant of the related dyad was housed solely with adults. Consequently, he may have had fewer options for social interactions, perhaps resulting in a higher concentration of his attention on the silverback. In turn, the social interactions of the silverback in this unit were more associated with encounters with the mother-infant dyad, which may have reduced the likelihood of agonistic encounters with the infant. If so, however, such influence from the mother's presence would not contradict expectations of kin selection nor reproductive strategy hypotheses, in that parental care and future reproductive possibilities could be intertwined [van Shaik and Paul, 1997].

Age presents yet another possible contributing factor to the observed differences, since the adult male of the unrelated pair was 17 years older than the silverback of the related dyad. Although an older male may be more experienced with immatures, it is equally possible that a younger silverback would be more playful and likely to interact with younger gorillas, as is seen in the case of the related dyad. Also, Hasani was 14 months younger than M'Bizi. However, given the data on wild mountain gorillas mentioned earlier [Fossey, 1979; Whitten, 1986], one would predict increased affiliative interactions between the silverback and the older infant if age were primarily responsible, but that is the reverse of what was observed in this study.

Finally, the observed differences could be a function of individuals' personalities and rearing histories. However, an important reminder is that the silverback in the unrelated dyad had a higher level of overall group social activity than the related silverback, and thus it would be difficult to argue that the related infant received more attention from the silverback simply because he was housed with a gorilla who had a "more interactive personality." With regard to rearing, all four gorillas have a history of at least a temporary separation from their natal groups during infancy. Such early experiences may have affected the gorillas' social development, but are extremely difficult to isolate and define in such a study.

Additional data would be helpful in reducing the number of possible interpretations of the results. Interestingly, a female sired by Frank was born into his group at the time of data collection, which could have provided critical information to contrast with the current data on his interactions with unrelated M'Bizi. Unfortunately for this study, the female infant and her mother were relocated shortly after birth, and they continue to be housed apart from Frank in an all-female group. Moreover, members of the related dyad have since been transferred to another zoo, precluding the opportunity for comparative data collection in this group.

In addition to the theoretical implications of kinship and male tolerance of infants, this study is relevant to the management of captive gorillas, which often requires the introduction of immatures into non-natal groups. Even if infants are introduced without incidence of attack or physical harm, there is still the possibility that their social development will be compromised. A close association between infants and adult males may create important opportunities for infants to observe and become familiarized with a wider range of adult social interactions [Watts and Pusey, 
1993]. For example, prolonged bouts of proximity and contact, which remained low within the unrelated dyad, can be crucial to infant socialization. This should be taken into account when managing gorillas in captivity, as social factors play a critical role in the overall development of the individual. Regardless, and importantly, the Lincoln Park Zoo has introduced both related and unrelated infants to the group silverback with great success.

\section{CONCLUSIONS}

1. Infants were the primary initiators of social encounters with their respective silverbacks, who exhibited low levels of active interest in the infants, as seen in wild mountain gorillas.

2. Differences between silverback-infant interactions within unrelated and related dyads were significant. The related silverback responded to his offspring with tolerance and affiliation, whereas obvious signs of disinterest and occasional intolerance characterized the relationship between the unrelated silverback and infant.

3. Data are consistent with a kin selection basis for male parental behavior and provide support for the theory that a male will establish stronger bonds with the offspring of females with whom he has had a strong association. These results also support the possibility that a male's parental behaviors may be indirectly intended to maintain his bond with the infant's mother, perhaps influencing female choice.

4. Although the data are consistent with a kin selection hypothesis, numerous alternative factors could contribute to the observed differences in these dyads.

5. Infants may be introduced into non-natal groups without being attacked or physically harmed by dominant males, but their subsequent relationships with these males may lack the close, affiliative interactions that enhance infant social development.

\section{ACKNOWLEDGMENTS}

This research was supported by a Dr. Scholl Science and Research Fellowship awarded to A.E. Enciso. We are especially indebted to Amy Rauhut for her time, effort, and guidance, and we also thank the staff of the Lincoln Park Zoo for helping to make this research possible. We are also very grateful for the excellent comments provided by two anonymous reviewers.

\section{REFERENCES}

Altmann J. 1974. Observational study of behavior: sampling methods. Behaviour 49:227-267.

Bennett C, Fried J. 1991. Dallas Zoo-gorilla ethology study. In: Gorilla Behavior Advisory Group, compilers. Compilation of gorilla ethograms. Atlanta: Atlanta/Fulton County Zoo. p 7-24.

DuBois T, Mead J, Cox C. 1991. Lowland gorillas: ethogram (Los Angeles Zoo). In: Gorilla Behavior Advisory Group, compilers. Compilation of gorilla ethograms. Atlanta: Atlanta/Fulton County Zoo. p 27-36.

Fossey D. 1979. Development of the mountain gorilla (Gorilla gorilla beringei): the first thirtysix months. In: Hamburg DA, McCown ER editors. The great apes. Menlo Park, CA: Benjamin/Cummings. p 139-184.

Gold KC. 1991. Gorilla development study. In: Gorilla Behavior Advisory Group, compilers. Compilation of gorilla ethograms. Atlanta: Atlanta/Fulton County Zoo. p 52-53.

Gold KC. 1993. Behavioral profiles of captive lowland gorillas: effects of age, sex, rearing history, and physical environment. Ph.D. Dissertation, Georgia Institute of Technology.

Harcourt AH. 1979. Social relationships between adult male and female mountain gorillas in the wild. Anim Behav 27:325-342.

Harcourt AH, Stewart KJ. 1981.Gorilla male relationships: can differences during immaturity lead 


\section{Enciso et al.}

to contrasting reproductive tactics in adulthood? Anim Behav 29:206-210.

Harcourt AH, Stewart KJ, Fossey D. 1981. Gorilla reproduction in the wild. In: Graham $\mathrm{C}$, editor. Reproductive biology of the great apes. New York: Academic Press. p 265-279.

Hoff MP, Nadler RD, Maple TL. 1981. Development of infant independence in a captive group of lowland gorillas. Dev Psychobiol 14:251-265.

Schaller GB. 1963. The mountain gorilla. Chicago: University of Chicago Press.

Stewart KJ, Harcourt AH. 1986. Gorillas: variation in female relationships. In: Smuts BB, Cheney DL, Seyfarth RM, Wrangham RW, Struhsaker TT, editors. Primate societies. Chicago: University of Chicago Press. p 155-164.

Tilford BL, Nadler RD.1978. Male parental behavior in a captive group of lowland gorillas (Gorilla gorilla gorilla). Folia Primatol 29: 218-228.

Trivers R. 1985. Social evolution. Menlo Park, CA: Benjamin/Cummings.
Tutin CEG. 1996. Ranging and social structure of lowland gorillas in Lopé Reserve, Gabon. In: McGrew WD, Marchant LF, Nishida T, editors. Great ape societies. Cambridge: Cambridge University Press. p 58-70.

van Shaik CP, Paul A. 1997. Male care in primates: does it ever reflect paternity? Evol Anthropol 5: 152-156.

Watts DP. 1996. Comparative socio-ecology of gorillas. In: McGrew WD, Marchant LF, Nishida T, editors. Great ape societies. Cambridge, Cambridge University Press. p 16-28.

Watts DP, Pusey AE. 1993. Behavior of juvenile and adolescent great apes. In: Pereira ME, Fairbanks LA, editors. Juvenile primates: lifehistory, development, and behavior. New York: Oxford University Press. p 148-167.

Whitten PL. 1986. Infants and adult males. In: Smuts BB, Cheney DL, Seyfarth RM, Wrangham RW, Struhsaker T, editors. Primate societies. Chicago: University of Chicago Press. p 343-357. 